\title{
X-rays from the eclipsing millisecond pulsar PSR J1740-5340 in the globular cluster NGC 6397
}

\author{
R. H. H. Huang and W. Becker
}

\begin{abstract}
Max-Planck-Institut für extraterrestrische Physik, Giessenbachstrasse 1, 85748 Garching, Germany
e-mail: rhuang@mpe.mpg.de
\end{abstract}

Received 10 August 2009 / Accepted 7 October 2009

\section{ABSTRACT}

\begin{abstract}
Aims. The millisecond pulsar PSR J1740-5340 in the globular cluster NGC 6397 shows radio eclipses over 40\% of its binary orbit. A first Chandra observation revealed indications for the X-ray flux being orbit dependent as well. In this work, we analysed five datasets of archival Chandra data taken between 2000 and 2007 to investigate the emission across the pulsar's binary orbit.

Methods. Utilizing archival Chandra observations of PSR J1740-5340, we performed a systematic timing and spectral analysis of this binary system.

Results. Using a $\chi^{2}$-test, the significance for intra-binary orbital modulation was found to be between $88.5 \%$ and $99.6 \%$, depending on the number of phase bins used to construct the light curve. Applying the unbiased statistical Kolmogorov-Smirnov (KS) test did not indicate any significant intra-binary orbital modulation. However, comparing the counting rates observed at different epochs, a flux variability on times scales of days to years is indicated. The possible origin of the X-ray emission is discussed in a number of different scenarios.
\end{abstract}

Key words. pulsars: individual: PSR J1740-5340 - stars: neutron - binaries: eclipsing - globular clusters: individual NGC 6397 X-rays: stars

\section{Introduction}

The millisecond pulsar (MSP) PSR J1740-5340 (6397A), located in the core-collapsed globular cluster NGC 6397 at a distance of $2.5 \mathrm{kpc}$, was discovered during a systematic search for ms-pulsars in Galactic globular clusters using the Parkes Radio Telescope (D'Amico et al. 2001a). It is orbiting around a massive late type companion ( $>0.14 M_{\odot}$; Orosz \& van Kerkwijk 2003) with an orbital period of 1.35 day (D'Amico et al. 2001b). The inclination of the binary system to the line of sight is $i \sim 50^{\circ}$ (Orosz \& van Kerkwijk 2003). The pulsar's spin period and period derivative are $P=3.65 \mathrm{~ms}$ (D'Amico et al. 2001a) and $\dot{P}=4.0 \times 10^{-20} \mathrm{~s} \mathrm{~s}^{-1}$ (Possenti et al. 2005). The contribution to $\dot{P}$ due to possible pulsar acceleration in the cluster's gravitational field is estimated to be smaller than $10^{-20} \mathrm{~s} \mathrm{~s}^{-1}$ by its large offset of 0.6 arcmin (corresponding to eleven core radii) from the cluster center (D'Amico et al. 2001b). The spin parameters imply a spin-down luminosity, a characteristic age and a dipole surface magnetic field of PSR J1740-5340 of $\dot{E} \sim 3.3 \times 10^{34} \mathrm{erg} \mathrm{s}^{-1}$, $\tau_{c}=P / 2 \dot{P} \sim 1.4 \times 10^{9}$ years, and $B_{\perp} \sim 3.9 \times 10^{8} \mathrm{G}$, respectively (Possenti et al. 2005).

The radio emission from PSR $\mathrm{J} 1740-5340$ is partially eclipsed in the orbital phase interval $0.05-0.45$ for approximately $40 \%$ of each orbit (D'Amico et al. 2001a,b). Moreover, strong fluctuations of the radio signals were observed at nearly all orbital phases (D'Amico et al. 2001b), which led to the interpretation that the pulsar is orbiting within an extended envelope of matter released from the companion. The optical light curve of the companion showing tidal distortions provides strong evidence that PSR J1740-5340 is orbiting a companion whose Roche lobe is nearly completely filled (Ferraro et al. 2001).
However, the system is in a radio-ejection phase in which accretion is inhibited by the radiation pressure exerted by the pulsar on the infalling matter. The strong interaction between the MSP flux and the plasma wind would explain the irregularities seen in the radio signals from PSR J1740-5340 (Ferraro et al. 2001; Burderi et al. 2002).

PSR J1740-5340 is in a wide orbit with a separation of $\sim 6.5 R_{\odot}$, so that the wind energy density impinging on the nondegenerate companion should be significantly less than that estimated for other much tighter eclipsing binary systems, such as the field ms-pulsar PSR B1957+20 which has a separation of $\sim 0.04 R_{\odot}$ from its companion (Arzoumanian et al. 1994). PSR J1740-5340 therefore is unlikely to drive a wind of sufficient density off its companion (D'Amico et al. 2001b). Orosz \& van Kerkwijk (2003) have studied the optical light curve of the companion and found no evidence of heating from the pulsar radiation, which supports the aforementioned inference. Sabbi et al. (2003) have investigated the chemical composition of the non-degenerate companion star and found a strong depletion of carbon. This suggests a scenario in which the companion is an evolved star that has lost most of its surface layers. In view of the high rotational velocity of the companion $\left(\sim 50 \mathrm{~km} \mathrm{~s}^{-1}\right.$; Sabbi et al. 2003), the stellar wind possibly can be strong enough to cause the mass-loss and result in an extended envelope of matter.

$\mathrm{X}$-ray emission from this binary system was detected with the Chandra X-ray Observatory (Grindlay et al. 2001). Based on an observation in 2000 (cf. Table 1) which only covered $\sim 40 \%$ of the binary orbit, Grindlay et al. (2002) reported evidence that the count rate of the system appears to increase by a factor of $\sim 2$ at phase 0.4 , just before the pulsar comes out of the radio eclipse. However, the short exposure time and the limited orbital 
Table 1. List of Chandra observations of PSR J1740-5340 in NGC 6397

\begin{tabular}{lccccccc}
\hline \hline Obs. date & Obs. ID & Instrument & Data mode & Effective exp. time & Orbital phase & Net count rate $^{a}$ & Off-axis(') $^{\prime}$ \\
\hline $2000-07-31$ & 79 & ACIS-I & Faint & $48.3 \mathrm{ks}$ & $0.146-0.553$ & $1.89 \pm 0.25^{b}$ & 0.8745 \\
$2002-05-13$ & 2668 & ACIS-S & Faint & $28.1 \mathrm{ks}$ & $0.035-0.267$ & $1.74 \pm 0.25$ & 0.9368 \\
$2002-05-15$ & 2669 & ACIS-S & Faint & $26.7 \mathrm{ks}$ & $0.501-0.719$ & $2.89 \pm 0.33$ & 0.9214 \\
$2007-06-22$ & 7461 & ACIS-S & Very Faint & $90.0 \mathrm{ks}$ & $0.189-0.950$ & $2.26 \pm 0.16$ & 0.3732 \\
$2007-07-16$ & 7460 & ACIS-S & Very Faint & $149.6 \mathrm{ks}$ & $0.437-1.714^{c}$ & $2.92 \pm 0.14$ & 0.2129 \\
\hline
\end{tabular}

Notes. ${ }^{(a)}$ ACIS-S net count rates in units of $10^{-3}$ counts/s corrected to the on-axis. ${ }^{(b)}$ The ACIS-I on-axis net count rate is $1.31 \pm 0.17$. In order to derive a comparable ACIS-S net count rate from the observed ACIS-I rate we convert the ACIS-I rate by using the WebPIMMS tool (http://heasarc.gsfc.nasa.gov/Tools/w3pimms.html). ${ }^{(c)}$ The total exposure time covers more than one binary orbit.

phase coverage of this first observation (cf. top panel in Fig. 1 and Table 1) did not support a detailed temporal and spectral analysis of the emission.

In this publication we report on a search for X-ray orbital modulation as well as on a spectral analysis of the PSR J17405340 binary system making use of archival Chandra data that cover various orbital phases ranges.

\section{Observations and data analysis}

In total, five observations were targeted on the PSR J17405340 binary system using the Chandra Advanced CCD Imaging Spectrometer (ACIS). The first observation was performed on 2000 July 31 using the front-illuminated (FI) ACIS-I3 chip, while the other four observations taken on 2002 May 13 and 15, 2007 June 26, and July 16 used the back illuminated (BI) chip ACIS-S3. We summarize the basic information of these observations in Table 1 . Their binary orbit coverages are shown in Fig. 1. As can be seen from this figure, only Obs. IDs 7460 and 7461 cover a larger fraction of the binary orbit. In the search for intra-orbital flux modulation and spectral variation, we therefore considered only these two datasets which have higher photon statistics and longer exposure times, while the other archival data were used to search for a possible flux variability on time scales of days or years. Data analysis was restricted to the energy range $0.3-8.0 \mathrm{keV}$. Searching for X-ray pulses from PSR J17405340 was precluded by the inappropriate temporal resolution of the observing modes used. Standard processed level-2 data were used. Correction for aspect offset was applied before analysis. All data were processed with Chandra Interactive Analysis Observations (CIAO) version 3.4 software and CALDB version 3.4.2.

\subsection{Search for orbital modulation}

In order to search for a modulation of the X-ray flux as a function of orbital phase, we extracted the photons from a circle of 2 arcsecond radius centered on the radio pulsar position RA $(\mathrm{J} 2000)=17^{\mathrm{h}} 40^{\mathrm{m}} 44^{\mathrm{s}} .59$, Dec $=-53^{\circ} 40^{\prime} 40^{\prime} \cdot 9$ (D'Amico et al. $2001 \mathrm{~b}$ ). In the 2007 observations, $\sim 90 \%$ of all source counts are located within this cut radius. The photon arrival times were corrected to the solar system barycenter with the CIAO tool AXBARY (JPL DE200 solar system ephemeris). We used the pulsar ephemeris from D'Amico et al. (2001b) and correct the photon arrival times for the orbital motion of the binary system (Blandford \& Teukolsky 1976). Since PSR J1740-5340 is far out of the globular cluster core, the correction for gravitational acceleration in the cluster potential was ignored.

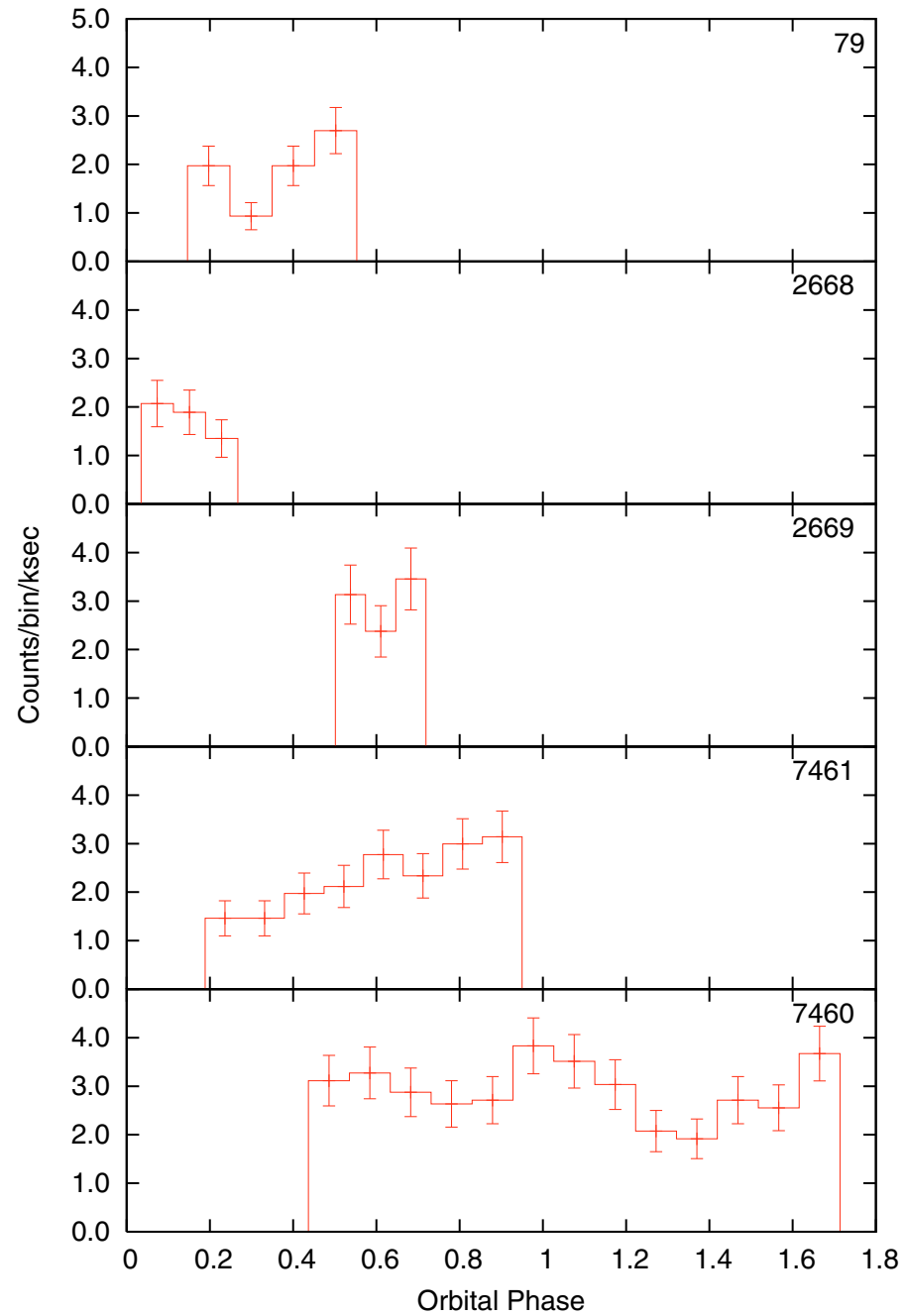

Fig. 1. Orbital phase coverages of five Chandra observations targeted on the binary system PSR J1740-5340. The $Y$-axis shows the X-ray source counts per bin per kilosecond in the energy range $0.3-8.0 \mathrm{keV}$. The $X$-axis is the binary orbital phase (from ephemeris of D'Amico et al. 2001b). $\phi=0.0$ is at the ascending node of the pulsar orbit. Binning is so that the signal-to-noise ratio in each bin is $\geq 3$. Error bars represent the $1 \sigma$ uncertainties. All X-ray light curves are corrected for vignetting and the various instrumental sensitivities of the different observing modes. Labels are the Obs. IDs (cf. Table 1).

We tested the 2007 data for intra-orbital flux modulation by fitting a constant to the X-ray light curve. In order to have the signal-to-noise ratio for each phase bin higher than $\sim 4$, the numbers of phase bins were restricted to be within 10 to 15 bins per 
Table 2. Significance for intra-orbital flux modulation by fitting the light curves of different phase bins against a constant by using the $\chi^{2}$ test statistics, including the best-fit mean levels.

\begin{tabular}{cccc}
\hline \hline Number of bins & Mean level & $\chi_{v}^{2}$ & Significance(\%) \\
\hline 10 & $37.1 \pm 2.7$ & 2.22 & 98.2 \\
11 & $33.4 \pm 2.4$ & 1.82 & 94.8 \\
12 & $29.6 \pm 2.1$ & 1.62 & 91.2 \\
13 & $28.6 \pm 2.1$ & 2.38 & 99.6 \\
14 & $26.4 \pm 2.0$ & 1.86 & 97.0 \\
15 & $24.7 \pm 1.9$ & 2.01 & 98.7 \\
20 & $18.4 \pm 1.1$ & 1.34 & 89.0 \\
40 & $9.2 \pm 0.5$ & 1.25 & 88.5 \\
\hline
\end{tabular}

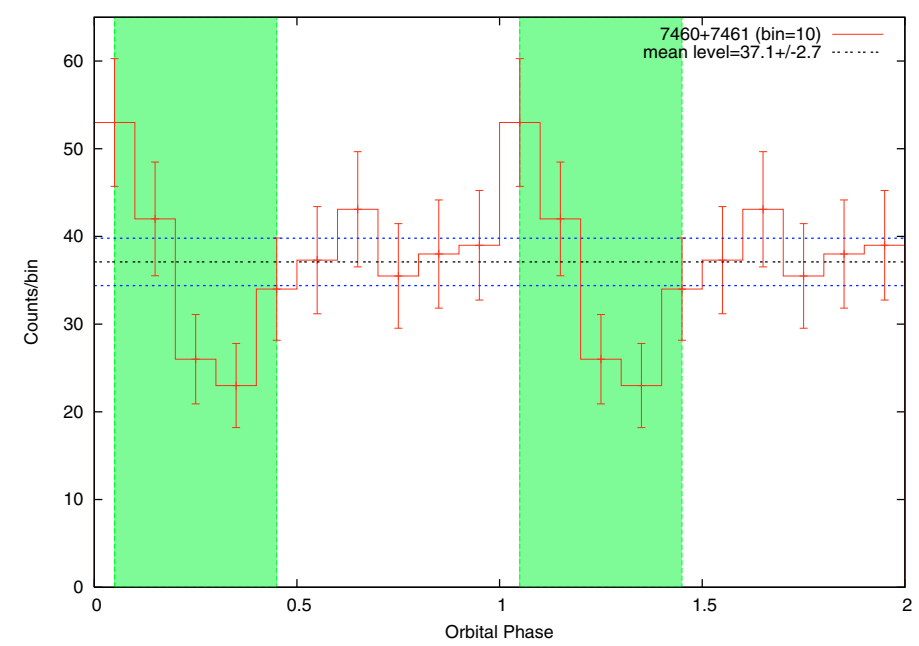

Fig. 2. X-ray source counts $(0.3-8.0 \mathrm{keV})$ vs. orbital binary phase according to the ephemeris of D'Amico et al. (2001b). Two orbital cycles are shown for clarity. The background noise level is found to be at $\sim 0.35$ counts/bin. $\phi=0.0$ corresponds to the ascending node of the pulsar orbit. Error bars indicate the $1 \sigma$ uncertainty. The shaded regions between the orbital phases $0.05-0.45$ and $1.05-1.45$ mark the radio eclipse of the pulsar PSR J1740-5340. The dotted lines indicates the best-fit constant level and its $1 \sigma$ uncertainty.

orbital period. In addition, we tested 20 and 40 bins per orbital period for comparison. Using a $\chi^{2}$-test, the significance for a flux modulation over the observed orbit was found to be between $88.5 \%$ and $99.6 \%$, depending on the number of phase bins used to construct the light curve. Table 2 summarizes the results for various bin numbers. The X-ray light curve for 10 phase bins is shown in Fig. 2.

The disadvantage of the $\chi^{2}$-test is its dependence on the number of phase bins. We therefore applied the KolmogorovSmirnov (KS) test to the unbinned light curve data in order to have a bin-independent statistical evaluation of the X-ray emission variability (see Fig. 3). Calculating the corresponding KS probabilities between our data set and the cumulative distribution function generated by assuming a constant X-ray flux indicated no significant deviation between these two distributions. The significance for an intra-orbital flux modulation from this test is only at the level of $\sim 68 \%$. A similar result is obtained if we restrict the analysis to the hard energy band above $2 \mathrm{keV}$.

\subsection{Spectral analysis}

To investigate whether the X-ray spectral behavior of PSR J1740-5340 varies across the orbit, we analysed the Xray spectrum within and outside the eclipsing region separately. The source and background spectra were extracted from the same region as mentioned in Sect. 2.1 and from a source-free region close to the pulsar. Response files for the corresponding observations were created by using the tools MKACISRMF and MKARF of CIAO. The background-subtracted count rates from the PSR J1740-5340 binary system per observation are given in Table 1. Extracted spectra were dynamically binned in accordance with the photon statistic in each dataset. Model spectra to Obs. IDs. 7460 and 7461 datasets were fitted using XSPEC 11.3.2. There are $183 \pm 14$ and $457 \pm 21$ net counts in total for the spectral analysis inside and outside the eclipsing region, respectively.

Assuming that the emission originates from the shock interaction of the pulsar wind with the wind of the companion star or from non-thermal emission processes in the pulsar magnetosphere, we expect the X-radiation to be synchrotron. To test this hypothesis, we fitted the spectrum with an absorbed power-law model (PL). For the PL fit, no significant variation of the spectral parameters is found between the spectrum inside and outside the eclipsing region. A thermal bremsstrahlung model (TB) was also tested, which physically implies that the X-ray emission is either from a hot plasma presented in the binary system or from the free-free radiation of the companion's corona. We also tested whether a single blackbody (BB) can provide an appropriate modeling of the data. However, this BB model cannot provide any statistically acceptable description of the observed spectra (i.e. $\chi_{v}^{2}>2$ ) either inside the eclipsing region or outside.

The spectral parameters inferred from these fits are summarized in Table 3. The spectral parameters inferred from the $\mathrm{PL} / \mathrm{TB}$ fits are found to be consistent with those reported by Grindlay et al. (2001, 2002). The best-fitted $N_{\mathrm{H}}$ for each spectrum varies marginally, but is consistent with the values of $\sim 1.03$ and $\sim 2.22 \times 10^{21} \mathrm{~cm}^{-2}$ inferred from the optical reddening of NGC 6397 and from the radio dispersion measure (DM) of PSR J1740-5340, respectively.

\section{Summary and discussion}

We have searched for the orbital modulation of the X-ray emission from PSR J1740-5340 associated with NGC 6397. Only one of five archival datasets cover a full binary orbit. Analysing this data with a Kolmogorov-Smirnov test revealed no significant intra-orbital flux modulation. However, correlating the ACIS-S vignetting-corrected net counting rates observed at various orbital phases in 2000, 2002 and 2007 and assuming that the system in general shows no intra-orbital flux modulation reveals a $\sim 3 \sigma$ flux variability on time scales of days to years (cf. Col. 7 in Table 1). Figure 4 depicts the ACIS-S net count rates observed in the various observations.

In the following, we will discuss three scenarios which we believe could be the main source of the observed X-rays in the PSR J1740-5340 binary system.

\section{The companion star}

The companion is observed to be a late-type star. It has an unusual position in the color-magnitude diagram (CMD) as being as luminous as a main-sequence turn-off star but redder (Ferraro et al. 2001; Orosz \& van Kerkwijk 2003). This makes it quite 
Table 3. Parameters from spectral fits to the PSR J1740-5340 binary system.

\begin{tabular}{lccccccc}
\hline \hline Orbital Phase & Model $^{a}$ & $N_{\mathrm{H}}{ }^{b}$ & $\Gamma / \mathrm{kT}(\mathrm{keV})$ & $\chi_{v}^{2} /$ d.o.f. & $F_{x}{ }^{c}$ & $L_{x}{ }^{d}$ & Note \\
\hline \multirow{2}{*}{$0.05-0.45$} & $\mathrm{PL}$ & $<1.4$ & $1.8_{-0.3}^{+0.4}$ & $1.39 / 20$ & $1.9_{-0.7}^{+1.8}$ & $1.4_{-0.5}^{+1.3} \times 10^{31}$ & inside eclipsing region \\
& $\mathrm{TB}$ & $<0.8$ & $7.2_{-3.4}^{+10.4}$ & $1.42 / 20$ & $1.7_{-0.4}^{+0.5}$ & $1.3_{-0.3}^{+0.4} \times 10^{31}$ & \\
\hline \multirow{2}{*}{$0.45-0.05$} & $\mathrm{PL}$ & $1.8_{-0.7}^{+1.0}$ & $1.7_{-0.2}^{+0.1}$ & $0.96 / 19$ & $3.3_{-0.8}^{+1.4}$ & $2.5_{-0.6}^{+1.1} \times 10^{31}$ & outside eclipsing region \\
& $\mathrm{TB}$ & $1.3_{-0.9}^{+0.6}$ & $7.3_{-1.7}^{+9.6}$ & $1.00 / 19$ & $2.9_{-0.5}^{+0.4}$ & $2.2_{-0.4}^{+0.3} \times 10^{31}$ & \\
\hline \multirow{2}{*}{$0.0-1.0$} & $\mathrm{PL}$ & $0.6_{-0.3}^{+0.4}$ & $1.5_{-0.1}^{+0.2}$ & $1.07 / 19$ & $2.8_{-0.8}^{+0.9}$ & $2.1_{-0.6}^{+0.7} \times 10^{31}$ & whole orbit \\
& $\mathrm{TB}$ & $<0.7$ & $16.1_{-7.6}^{+23.2}$ & $1.11 / 19$ & $2.6_{-0.4}^{+0.3}$ & $1.9_{-0.3}^{+0.2} \times 10^{31}$ & \\
\hline
\end{tabular}

Notes. Column 1 gives the orbital phase, Cols. 2-5 show the spectral model, the hydrogen column density $N_{\mathrm{H}}$, the best-fitted photon index or temperature, and the reduced $\chi^{2}$ together with degrees of freedom. Cols. 6-7 list the unabsorbed X-ray flux and luminosity in the $0.3-8.0 \mathrm{keV}$ energy range. Quoted errors indicate the $68 \%$ confidence level for one parameter of interest.

${ }^{(a)} \mathrm{PL}=$ power law; TB = thermal bremsstrahlung. ${ }^{(b)}$ In units of $10^{21} \mathrm{~cm}^{-2}$. ${ }^{(c)}$ Unabsorbed X-ray flux in units of $10^{-14} \mathrm{erg} \mathrm{cm}^{-2} \mathrm{~s}^{-1}$ and in the energy range of $0.3-8.0 \mathrm{keV} .{ }^{(d)}$ Unabsorbed X-ray luminosity in units of erg s ${ }^{-1}$ and in the energy range of $0.3-8.0 \mathrm{keV}$ for a distance of $2.5 \mathrm{kpc}$ (Grindlay et al. 2001).

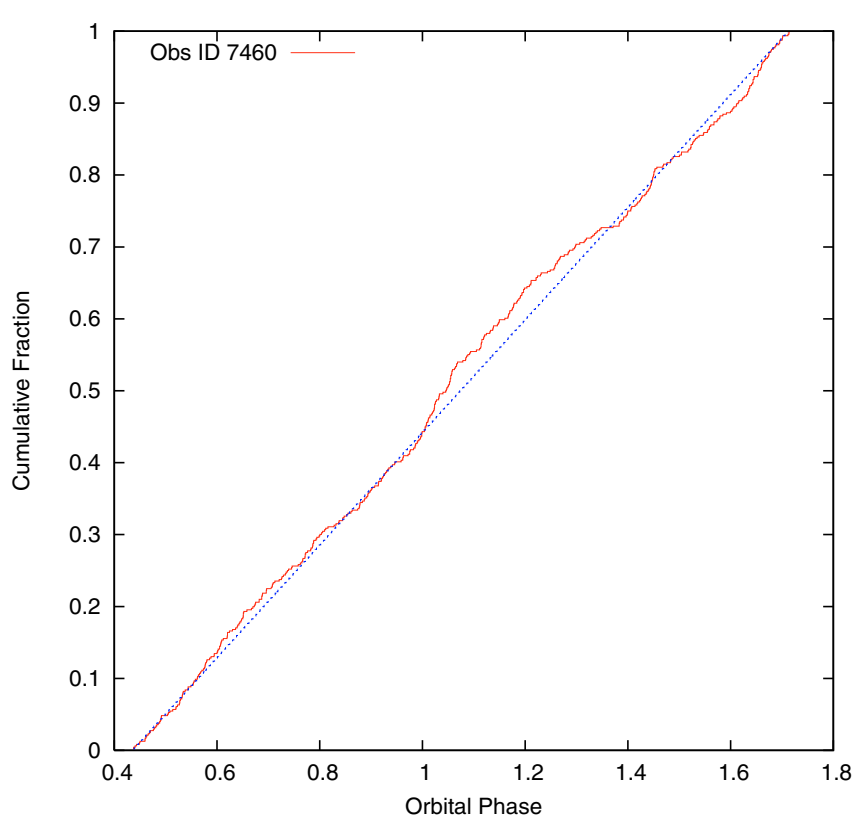

Fig. 3. Kolmogorov-Smirnov test. The cumulative distribution function from the observed data is plotted in red and the blue dotted line shows the estimated cumulative distribution generated by assuming a constant $\mathrm{X}$-ray flux.

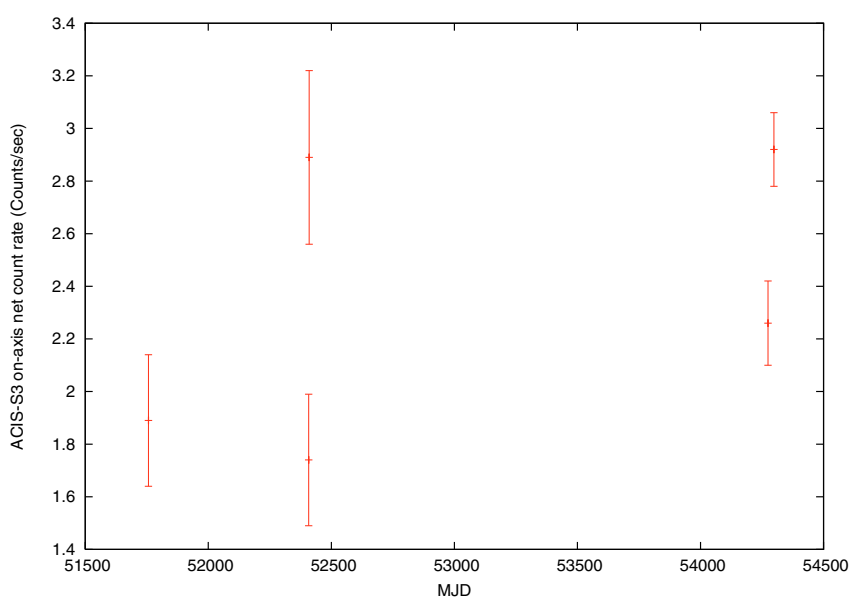

Fig. 4. ACIS-S3 count rates of the PSR J1740-5340 binary system vs. observation dates. similar to the "red straggler" active binaries which are identified to be X-ray sources in other globular clusters (Albrow et al. 2001; Edmonds et al. 2003a,b; Bassa et al. 2008). Detailed studies of X-ray sources in various globular clusters by Bassa et al. (2004) and Verbunt et al. (2008) have shown that the separatrix $\log L_{0.5-2.5 \mathrm{keV}}=34.0-0.4 M_{V}$ allows one to distinguish between the population of cataclysmic variables and that of magnetically active binaries. Here, $M_{V}$ is the absolute $V$-band magnitude, which for the PSR J1740-5340 binary system was observed to be $M_{V} \sim 5$ (Orosz \& van Kerkwijk 2003). Taking this together makes the properties observed from the companion star consistent with that from rapid rotating stars. The X-ray luminosities of late-type stars (i.e. F7 to M5) are found to be well correlated with their equatorial rotational velocities $v_{\text {rot }}$, according to $L_{\mathrm{X}} \simeq 10^{27} v_{\text {rot }}^{2} \mathrm{erg} \mathrm{s}^{-1}$ (Pallavicini et al. 1981). Adopting the $v_{\text {rot }} \sim 50 \mathrm{~km} \mathrm{~s}^{-1}$ as reported by Sabbi et al. (2003) yields an expected X-ray luminosity of $\sim 2.5 \times 10^{30} \mathrm{erg} \mathrm{s}^{-1}$. Given that the $L_{\mathrm{X}}$ vs. $v_{\text {rot }}^{2}$ correlation has a scatter of one order of magnitude (Pallavicini et al. 1981) it cannot be excluded that the X-ray emission observed from the PSR J1740-5340 binary is coronal emission from the companion star only. No significant X-ray intra-orbital modulation is observed, which is unlike what we have seen in the optical bands. The optical emission is expected to come from the surface of the companion star while the X-ray emission comes from the corona. As the companion star is interpreted to be tidal-distorted (Orosz \& van Kerkwijk 2003), the emitting area responsible for the optical intensity appears to be modulated. However, the X-ray emission is coronal and may be less or not affected by this distortion.

\section{The millisecond pulsar}

Considering a possible contribution from the pulsar magnetosphere is justified by the pulsar's spin-down energy of $\dot{E} \sim$ $3.3 \times 10^{34} \mathrm{erg} \mathrm{s}^{-1}$. By assuming the best-fit X-ray conversion efficiency found for other field and millisecond pulsars, $L_{\mathrm{X}} \sim 10^{-3}-10^{-4} \dot{E}$ (Becker 2009), the spin-down power of PSR J1740-5340 implies an X-ray luminosity which is of the order of $\sim 10^{30}-10^{31} \mathrm{erg} \mathrm{s}^{-1}$, in agreement with the observed luminosity (cf. Table 3). Observing the pulsar in a mode suitable to resolve its X-ray pulses would be worthwhile and could constrain this interpretation. 


\section{Intrabinary shock emission}

Grindlay et al. (2002) suggested that the X-ray emission they detected from the PSR J1740-5340 binary system is due to the interaction between the pulsar and shocked gas lifted from the stellar companion. In the shock, the extended matter in the orbit is compressed and gives rise to a power law distribution of synchrotron-emitting particles, $N(\gamma) \propto \gamma^{-p}$, by accelerating a fraction of these particles to very high energies, where $\gamma$ is the Lorentz factor of the emitting particles. This should lead to an enhancement of X-ray emission from the system as the pulsar enters the eclipsing region and a subsequent decrease due to the increased absorption in this region. Cheng et al. (2006) found that the X-ray luminosity of intrabinary shock emission in the $2-10 \mathrm{keV}$ band can be described by $L_{\mathrm{X}}^{\mathrm{th}} \sim(0.3-1.2) \times$ $10^{32} D_{11}^{-(p-1) / 2} \dot{E}_{35}^{(p+5) / 4} \mathrm{erg} \mathrm{s}^{-1}$, assuming a Lorentz factor for the synchrotron-emitting particles of $\gamma=(0.5-1.0) \times 10^{6}$, the fractional energy density of electrons to be $\epsilon_{e}=0.5$ and the fractional energy density of the magnetic field to be $\epsilon_{B}=0.02-0.1$. $p$ and $D_{11}$ denote the index of the postshock electron energy distribution $N(\gamma) \propto \gamma^{-p}$, and the orbital separation in units of $10^{11} \mathrm{~cm}$. The X-ray photon index inferred from the spectrum is $\Gamma \sim 1.8$ which would suggest that the emission from the shock is in a slow cooling regime (Cheng et al. 2006). The photon index is related to $p$ as $\Gamma=(p+1) / 2$. The observed $\Gamma$ implies that $p$ is $\sim 2.6$. With $\dot{E}_{35}=0.33$ and $D_{11}=4.5, L_{\mathrm{X}}^{\text {th }}$ is found to be in the range of $\sim(0.1-4.4) \times 10^{30} \mathrm{erg} \mathrm{s}^{-1}$, which is at the observed level. Intrabinary shock emission thus can explain the observed X-ray luminosity though the modulation depth and amplitude, which is also a function of the inclination angle, is unconstrained from this model.

Based on the preceding discussion, it is not possible to firmly distinguish the possible emission scenarios which may, alone or in combination, be responsible for the observed X-ray emission. To disentangle them would require detailed orbital-phase resolved spectroscopy along with a search for X-ray pulses from the millisecond pulsar. Further dedicated X-ray observations covering more binary orbits are certainly needed to further constrain the emission processes at work in this binary system.

Acknowledgements. This work made use of the Chandra data archive. The first author thanks C.Y. Hui and M. Güdel for providing helpful suggestions and also acknowledges the receipt of funding provided by the Max-Planck Society in the frame work of the International Max-Planck Research School on Astrophysics at the University of Munich.

\section{References}

Albrow, M. D., Gilliland, R. L., Brown, T. M., et al. 2001, ApJ, 559, 1060 Arzoumanian, Z., Fruchter, A. S., \& Taylor, J. H. 1994, ApJ, 426, L85 Bassa, C., Pooley, D., Homer, L., et al. 2004, ApJ, 609, 755

Bassa, C. G., Pooley, D., Verbunt, F., et al. 2008, A\&A, 488, 921

Becker, W. 2009, in Astrophysics and Space Science Library, 357, ed. W. Becker, 91

Blandford, R., \& Teukolsky, S. A. 1976, ApJ, 205, 580

Burderi, L., D’Antona, F., \& Burgay, M. 2002, ApJ, 574, 325

Cheng, K. S., Taam, R. E., \& Wang, W. 2006, ApJ, 641, 427

D’Amico, N., Lyne, A. G., Manchester, R. N., Possenti, A., \& Camilo, F. 2001a, ApJ, 548, L171

D’Amico, N., Possenti, A., Manchester, R. N., et al. 2001b, ApJ, 561, L89

Edmonds, P. D., Gilliland, R. L., Heinke, C. O., \& Grindlay, J. E. 2003a, ApJ, 596,1177

Edmonds, P. D., Gilliland, R. L., Heinke, C. O., \& Grindlay, J. E. 2003b, ApJ, 596, 1197

Ferraro, F. R., Possenti, A., D’Amico, N., \& Sabbi, E. 2001, ApJ, 561, L93

Grindlay, J. E., Camilo, F., Heinke, C. O., et al. 2002, ApJ, 581, 470

Grindlay, J. E., Heinke, C. O., Edmonds, P. D., Murray, S. S., \& Cool, A. M. 2001, ApJ, 563, L53

Orosz, J. A., \& van Kerkwijk, M. H. 2003, A\&A, 397, 237

Pallavicini, R., Golub, L., Rosner, R., et al. 1981, ApJ, 248, 279

Possenti, A., D'Amico, N., Corongiu, A., et al. 2005, in Binary Radio Pulsars, ed. F. A. Rasio \& I. H. Stairs, ASP Conf. Ser., 328, 189

Sabbi, E., Gratton, R. G., Bragaglia, A., et al. 2003, A\&A, 412, 829

Verbunt, F., Pooley, D., \& Bassa, C. 2008, in IAU Symp., 246, 301 